\title{
Clinical Experience with a High Precision Image-Guided Neurosurgery System ${ }^{\star}$
}

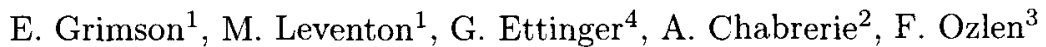 \\ , S. Nakajima ${ }^{2}$, H. Atsumi ${ }^{3}$, R. Kikinis ${ }^{2}$, and P. Black ${ }^{3}$ \\ 1 MIT AI Laboratory, Cambridge MA, USA \\ welg@ai.mit.edu \\ 2 Radiology, Brigham \& Womens Hospital, Harvard Medical School, Boston USA \\ 3 Neurosurgery, Brigham \& Womens Hospital, Harvard Medical School, Boston USA \\ 4 Alphatech, Burlington MA USA
}

\begin{abstract}
We describe an image-guided neurosurgery system which we have successfully used on 70 cases in the operating room. The system is designed to achieve high positional accuracy with a simple and efficient interface that interferes little with the operating room's usual procedures, but is general enough to use on a wide range of cases. It uses data from a laser scanner or a trackable probe to register segmented MR imagery to the patient's position in the operating room, and an optical tracking system to track head motion and localize medical instruments. Output visualizations for the surgeon consist of an "enhanced reality display," showing location of hidden internal structures, and an instrument tracking display, showing the location of instruments in the context of the MR imagery. Initial assessment of the system in the operating room indicates a high degree of robustness and accuracy.
\end{abstract}

\section{Introduction}

Many surgical procedures require highly precise localization, often of deeply buried structures, in order for the surgeon to extract targeted tissue with minimal damage to nearby structures. While methods such as MRI and CT are valuable for imaging and displaying the internal 3D structure of the body, the surgeon must still relate what he sees on the 3D display with the actual patient.

Traditional clinical practice often only utilizes $2 \mathrm{D}$ slices of MR or CT imagery, requiring the surgeon to mentally transform that information to the actual patient, thus there is a need for techniques to register $3 \mathrm{D}$ reconstructions of internal anatomy with the surgical field. Such image-guided surgical tools allow the surgeon to directly visualize important structures, and plan and act accordingly. Visualization methods include "enhanced reality visualization" [11], in which rendered internal structures are overlaid on the surgeon's field-of-view, and instrument tracking, in which medical instruments acting on the patient are localized and visualized in the 3D MR or CT imagery.

\footnotetext{
* This report describes research supported in part by DARPA under ONR contract
} N00014-94-01-0994. 

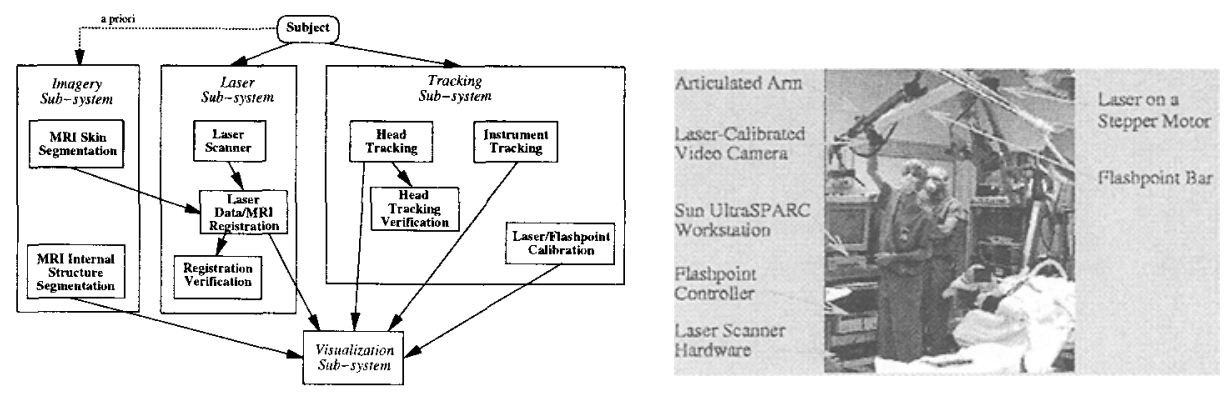

Fig. 1. (left) Image-guided surgery system architecture. (right) Setup of system in operating room.

The key components of an accurate, reliable, image-guided surgery system are: creating accurate, detailed, patient-specific models of relevant anatomy for the surgical procedure; registering the models, and the corresponding imagery, to the patient; maintaining the registration throughout the surgical procedure; and tracking medical instruments in the surgical field in order to visualize them in the context of the MR/CT imagery and the reconstructed models.

We have developed a system which addresses these issues, primarily in neurosurgery. We had earlier reported our registration algorithms [11], and algorithmic testing of the system [10]. The important developments we report here are integration of tracking techniques, engineering of the system into an effective surgery tool, evaluation of the system's performance under control conditions, and initial experience of using the system on 70 cases in the operating room.

\section{Image-Guided Neurosurgery System}

Neurosurgery is an ideal application for image-guided techniques, by virtue of the high precision it requires, the need to visualize nearby tissue, the need for planning of optimal trajectories to target tissue, and the need to localize visually indistinguishable, but functional different, tissue types. Early attempts to achieve image-guided neurosurgery consisted of stereotactic frames which directly provided the fiducials for registering the MRI or CT data to the patient [8]. More recently, frameless stereotaxy systems have been pursued by many groups $[1,2,5,15,18,23]$, and usually consist of two components: registration and tracking. We have added a third, initial, component to our system - reconstructed models of the patient's anatomy. The system's components are described below.

The architecture of our image-guided surgery system (Figure 1) supports frameless, non-fiducial, registration of medical imagery by matching surface data, and supports optical tracking of patient and instrument locations. The system (Figure 1) consists of a portable cart containing a Sun UltraSPARC workstation and the hardware to drive the laser scanner and Flashpoint tracking system. On 
top of the cart is mounted an articulated extendable arm to which we attach a bar housing the laser scanner and Flashpoint cameras. The three linear Flashpoint cameras are inside the bar. The laser is attached to one end of the bar, and a video camera to the other. The joint between the arm and scanning bar has three degrees-of-freedom to allow easy placement of the bar in desired configurations.

\subsection{Imagery Sub-system}

MRI is the prime imaging modality for the neurosurgery cases we support. The images are acquired prior to surgery without a need for special landmarking strategies. To process the imagery, a wide range of methods (e.g., $[25,21,16,19$, 22]) have been applied to the segmentation problem, i.e. identifying different tissue types in medical imagery. Our current approach to segmentation uses an automated method to initially segment into major tissue classes while removing gain artifacts from the imager $[25,13]$, then uses operator driven interactive tools to refine this segmentation. This latter step primarily relies on 3D visualization and data manipulation techniques to correct and refine the initial automated segmentation. The segmented tissue types include skin, used for registration, and internal structures such as brain, tumor, vessels, and ventricles. These segmented structures are processed by the Marching Cube algorithm [14] to construct isosurfaces and support surface rendering for visualization.

The structural models of patients constructed using such methods can be augmented with functional information. For example, functional MRI methods or transcranial magnetic stimulation methods [7] can be used to identify motor or sensory cortex. This data can then be fused with the structural models [26] to augment such models. In each case, segmentation produces information in a local coordinate frame, which must be merged together. We currently use a registration method based on Mutual Information [26] to do this. The result is an augmented, patient-specific, geometric model of relevant structural and functional information.

\subsection{Registration Sub-system}

Registration is the process by which the MRI or CT data is transformed to the coordinate frame of the patient. The most common form of registration uses fiducials $[1,15,20,23\}$ : either markers attached to the skin or bone prior to imaging or anatomically salient features on the head. The fiducials are manually localized in both the MR or CT imagery and on the patient and the resulting correspondences are used to solve for the registration. Fiducial systems may not be as accurate as frame-based methods-Peters [17] reports fiducial accuracy about an order of magnitude worse than frame-based methods, but Maciunas [15] reports high accuracy achieved with novel implantable fiducials.

Another registration approach is surface alignment in which the MRI skin surface is aligned with the patient's scalp surface in the operating room. Ryan [18] generates the patient's scalp surface by probing about 150 points with a trackable medical instrument. Colchester [5] uses an active stereo system to 

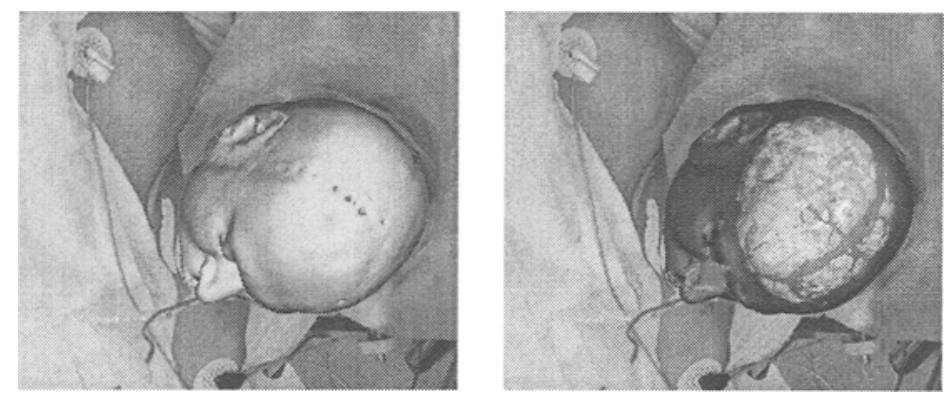

Fig. 2. (left) Initial alignment of laser and MRI data, showing overlay of MRI skin data on laser scanner's video image. (right) Enhanced reality visualization of the patient showing hidden internal structures overlaid on the surgeon's view of the patient.

construct the scalp surface. We also perform the registration using surface alignment [9], benefiting from its dense data representation, but use either a laser scanner to construct the patient's scalp surface or a trackable probe to obtain data points from the patient's skin surface for registration.

We have used two related methods to register the reconstructed model to the actual patient position. In the first method, we use a laser scanner to collect $3 \mathrm{D}$ data of the patient's scalp surface as positioned on the operating table. The scanner is a laser striping triangulation system consisting of a laser unit (low power laser source and cylindrical lens mounted on a stepper motor) and a video camera. The laser is calibrated a priori by using a calibration gauge of known dimensions to calculate the camera parameters and the sweeping angles of the laser. In the operating room the laser scanner is placed to maximize coverage of the salient bony features of the head, such as nose and eye orbits. To ensure accurate registration we can supplement the laser data with points probed with a Flashpoint pointer, similar to [18], to include skin points that are not visible to the laser in the registration. The acquired laser data is overlaid on the laser scanner's video image of the patient for specification of the region of interest. This process uses a simple mouse interface to outline the region of the head on which we want to base the registration. This process need not be perfect- the registration is designed to deal robustly with outliers. The laser scan takes about 30 seconds once the sensor is appropriately placed above the patient.

An alternative method is to simply use a trackable probe to acquire data. In this case, we trace paths on the skin of the patient with the trackable probe, recording positional information at points along each path. These points are not landmarks, but simply replace the lines of laser data. The registration process is the same, whether matching laser data or trackable probe data to the skin surface of the MRI model.

One of the keys to our system is the integration of a reliable and accurate data-to-MRI registration algorithm. Our registration process is described in detail in [11]. It is a three step process performing an optimization on a six parameter rigid transformation, which aligns the data surface points with the MRI 
skin surface. The steps consist of: (1) A manual initial alignment in which we roughly align the two surfaces. Accurate manual alignment can be very difficult, but we aim only to be within $20^{\circ}$ of the correct transformation which subsequent steps solve for. This process is performed using two displays and takes about 60 seconds. In one display, the rendered MRI skin is overlaid on the laser scanner's video view of the patient, and the MRI data is rotated and translated in $3 \mathrm{D}$ to achieve a qualitatively close alignment. In the second display, the laser data is projected onto three orthogonal projections of the MRI data. The projected MRI data is colored such that intensity is inversely proportional to distance from the viewer. In each overlay view, the laser data may be rotated and translated in $2 \mathrm{D}$ to align the projections. An alternative to manual initial alignment is to record three known points using the trackable probe (e.g. tip of the nose, tip of the ear), then identify roughly the same point in the MRI model. This process determines a rough initial alignment of the data to the MR reconstruction, and typically takes less then 5 seconds. (2) Automated interpolated alignment which performs optimization over a large region of convergence [9-11]. This process runs in about 10 seconds on a Sun UltraSPARC workstation. The method basically solves for the transform that optimizes a Gaussian weighted least-squares fit of the two data sets. (3) Automated detailed alignment which performs optimization to accurately localize the best surface data to MRI transformation[9-11]. This process runs in about 10 seconds on a Sun UltraSPARC workstation. The method basically solves a truncated least-squares fit of the two data sets, refining the transformation obtained in the previous step.

Three verification tools are used to inspect the registration results as the objective functions optimized by the registration algorithm may not be sufficient to guarantee the correct solution. One verification tool overlays the MRI skin on the video image of the patient, (Figure 2), except that we animate the visualization by varying the blending of the MRI skin and video image. A second verification tool overlays the sensed data on the MRI skin by color-coding the sensed data by distance between the data points and the nearest MRI skin points. Such a residual error display identifies possible biases remaining in the registration solution. A third verification tool compares locations of landmarks. Throughout the surgery, the surgeon uses the optically tracked probe to point to distinctive anatomical structures. The offset of the probe position from the actual point in the MR volume is then observed in the display.

\subsection{Tracking Sub-system}

Tracking is the process by which objects are dynamically localized in the patient's coordinate system. Of particular interest to us is the tracking of medical instruments and the patient's head. Optical trackers use multiple cameras to triangulate the 3D location of flashing LEDs that may be mounted on any object to be tracked. Such devices are generally perceived as the most accurate, efficient, and reliable localization system $[2,4]$. Other methods, such as acoustic or magnetic field sensing are being explored as well, but tend to be more sensitive 

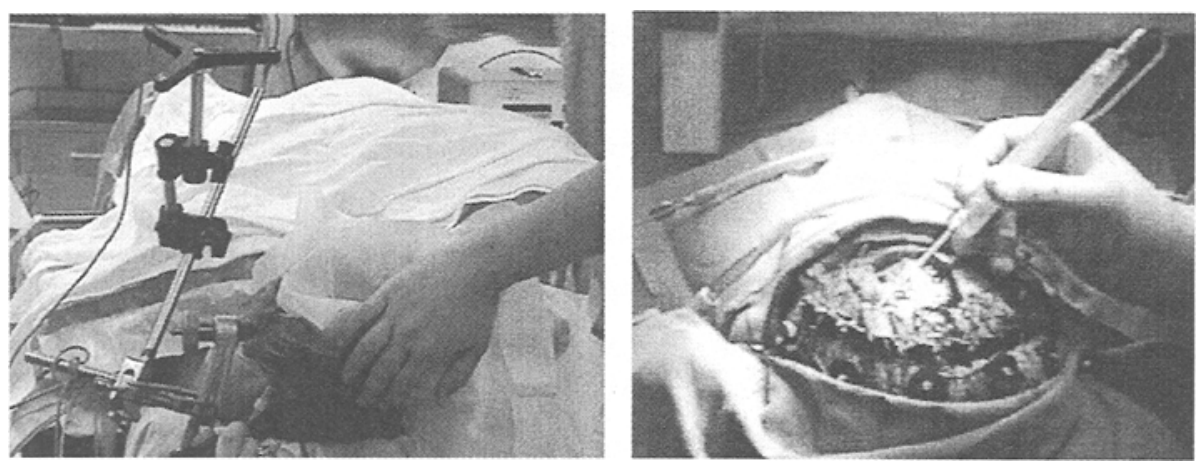

Fig. 3. Trackable configuration of LEDs attached to head clamp, or to the skin flap.

to environmental effects. We use optical tracking, the Flashpoint system by IGT Inc., Boulder, CO, USA, due to its accuracy and ease-of-use benefits.

Tracking patient head motion is often necessary since the head is not always clamped to the operating table, the head may move relative to the clamp, the operating table may be moved, or the hardware performing the tracking may be moved to rearrange lights or other equipment in the operating room. Although not all image-guided surgery systems account for patient motion, $[1,2,5,15,18]$ solve this problem by attaching trackable markers to the head or clamp. We also currently utilize an optically trackable configuration of markers attached to a Mayfield clamp (Figure 3). We have also experimented with directly attaching trackable LEDs to the skin surface of the patient. Our experience is that while in most cases this worked well, it required that the surgeon carefully plan the location of the LEDs to ensure that they did not move between initial placement and opening of the skin flap.

We require direct line-of-sight from the Flashpoint cameras to the LEDs at times when the surgeon requires image-guidance. In order to maintain such lineof-sight we can re-locate the scanning bar such that it is both out of the way of the surgeon but maintains visibility of the LEDs. Such dynamic reconfiguration of the scanning bar is a benefit of the head tracking process.

Instrument tracking is performed by attaching two LEDs to a sterile pointer. The two LEDs allow us to track the 3D position of the tip of the pointer as well as its orientation, up to the twist angle which is not needed for this application. Figure 3 shows the surgeon using the trackable pointer in the opened craniotomy.

\subsection{Visualization Sub-system}

Two types of visualizations are provided to the surgeon on the workstation monitor. One is an enhanced reality visualization in which internal structures are overlaid on the video image of the patient. The video image is set up to duplicate the surgeon's view of the patient. Any segmented MR structures may be displayed at varying colors and opacities (see Figure 2). 

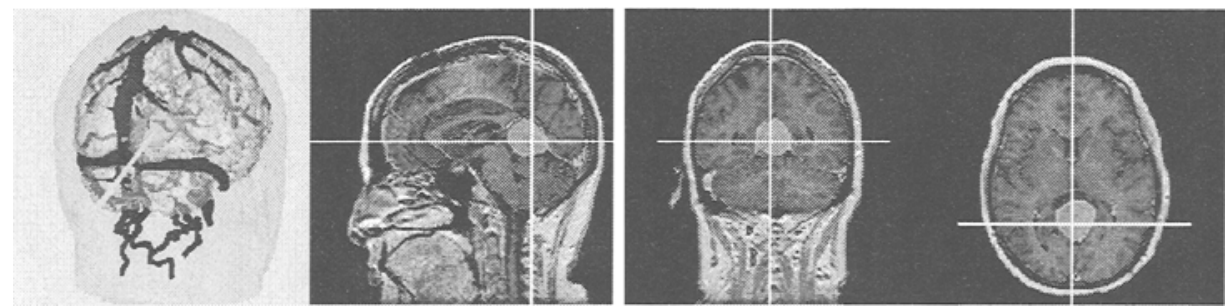

Fig. 4. Pointer tracking in 3D MRI rendering and three orthogonal MRI slices.

A second visualization shows the location of the pointer tip in a $3 \mathrm{D}$ rendering of selected MRI structures and in three orthogonal MRI slices (see Figure 4). These visualizations are updated twice per second as the pointer is moved.

\section{Operating Room Procedure}

Using our system, as seen from the surgeon's perspective, involves: (1) Prepare patient for surgery as per usual procedure including clamping the head. Head is still visible. (2) Attach a configuration of LEDs to the head clamp, and record the positions of the LEDs in the Flashpoint system. (3) Register MRI to patient by placing our scanner bar over patient's head. The bar is generally about $1.5 \mathrm{~m}$ away from head. Scan patient's head by swabbing a trackable probe across the skin. Typically several swabs are used, designed to cover a wide range of positions on the patient. It is often convenient to include swabs along known paths such as across the cheeks or down the nose, as these paths will aid in inspecting the resulting registration. (4) The Flashpoint/laser bar may be re-positioned at any point to avoid interference with equipment and to maintain visibility of LEDs. (5) Sterilize and drape patient. Any motion of the patient during this process will be recorded by movements of the LED configuration attached to the head clamp. (6) Proceed with craniotomy and surgical procedure. (7) At any point, use sterile Flashpoint pointer to explore structures in the MR imagery.

\section{Performance Analysis}

To evaluate the performance of our registration and tracking subsystems, we have performed an extensive set of controlled perturbation studies [6]. In these studies, we have taken existing data sets, simulated data acquisition from the surface of the data, added noise to the simulated surface data, then perturbed the position of data and solved for the optimal registration. Since we know the starting point of the data, we can measure the accuracy with which the two data sets are re-registered.

While extensive details of the testing are reported in [6], the main conclusions of the analysis are: 

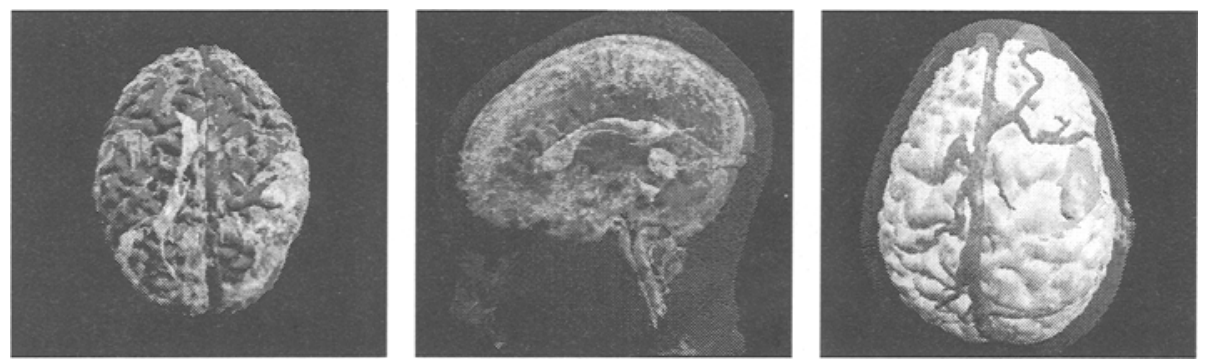

Fig. 5. Examples of neurosurgical cases. The last example includes a fusion of $\mathrm{fMR}$ data overlaid on top of the structural model.

- accurate and stable registration is achieved for up to 45 degree rotational offsets of the data sets, with other perturbations;

- accurate and stable registration is achieved for up to 75 degree rotational offsets of the data sets, with no other perturbations;

- robust registration is obtained when the surface data spans at least $40 \%$ of the full range of the surface, and is generally obtained with as little as $25 \%$ coverage;

- small numbers of outliers do not affect the registration process.

\section{Operating Room Results}

We have used the described image-guided neurosurgery system on 70 patients. These cases included: 44 Supratentorial - 22 high grade, 22 low grade; 6 Meningiomas; 3 Metastases; 2 Posterior Fossa; 1 Meningioangiomatosis; 7 Intractable Epilepsy; 4 Vascular; 2 Biopsies; and 1 Demyelinating lesion.

In all cases the system effectively supported the surgery:

- by providing guidance in planning bone cap removal - this was done through the augmented reality visualization in which the surgeon could visualize paths to the critical tissue and plan an appropriate entry point.

- identifying margins of tumor - this was done by tracing the boundaries of tissue with the trackable probe.

- localizing key blood vessels.

- and orienting the surgeon's frame of reference.

Selected examples are shown in Figure 5.

To qualitatively validate the system's performance, the surgeon placed the pointer on several known landmarks: skull marks from previous surgeries, ventricle tip, inner skull bones such as eye orbits, sagittal sinus, and small cysts or necrotic tissues. He then estimated their position in the MRI scan, and we compared the distance between the expected position and the system's tracked 

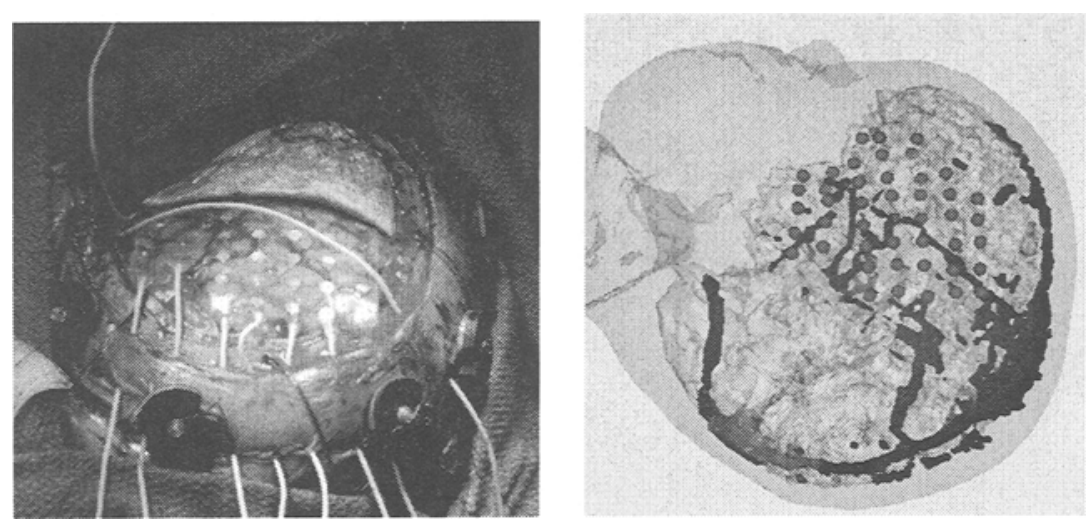

Fig. 6. Grid of electrodes placed on cortical surface. Location of grid points overlaid onto MR reconstruction, with focal area highlighted.

position. In all cases, this error was less than two voxels (MRI resolution was $0.9375 \mathrm{~mm} \times 0.9375 \mathrm{~mm} \times 1.5 \mathrm{~mm}$ ).

One example of the effectiveness of the system is illustrated by the following study. Twenty patients with low-grade gliomas underwent surgery with the system. The pathologies included 10 low grade astrocytomas (grades I, II out of IV), 7 oligoastrocytomas (without anaplastic features) and 3 oligodendrogliomas. Thirteen patients underwent cortical mapping including 7 who underwent speech and motor mapping, 2 motor alone, 1 speech alone and 3 motor and sensory. $31 \%$ had a subtotal resection, the remainder had total resection. One patient exhibited temporary left-sided weakness. Cortical mapping had represented the sensory cortex diffusely behind this patient's gross tumor. The post-operative weakness was temporary and was thought to be due to swelling. One patient showed a mild, left upper extremity proprioreceptive deficit which was due to a vascular accident on post-operative day one. The remaining patients were neurological intact following the procedure.

In addition to the tumor resection cases, we have also used the system in 10 pediatric epilepsy cases [3]. In the first stage of this two stage surgery, the patient's cortex is exposed and a grid of electrical pickups is placed on the cortical surface. A lead from each pickup is threaded out through the skin for future monitoring. In addition to registering the MRI model of the patient to his/her position, the location of each electrical contact is recorded and transformed to MRI coordinates. The patient is then closed up and monitored for several days. During any seizure event, the activity from each cortical probe is monitored, and transformed to the MRI model. This enables the surgeon to isolate potential foci in MRI coordinates. During a second surgical procedure, the augmented MRI model is reregistered to the patient and the locations of the hypothesized foci are presented to the surgeon for navigational guidance. An example of this is shown in Figure 6. 
To see the range of cases handled by our system, we encourage readers to visit the web site:

http://splweb. bwh .harvard . edu: 8000/pages/comonth. html

which shows selected cases with descriptions of the use and impact of the navigation system on the case.

\section{Summary}

We have described an image-guided neurosurgery system, now in use in the operating room. The system achieves high positional accuracy with a simple, efficient interface that interferes little with normal operating room procedures, while supporting a wide range of cases. Qualitative assessment of the system in the operating room indicates strong potential. In addition to performing quantitative testing on the system, we are also extending its capabilities by integrating a screw-based head tracking system and improved visualization capabilities.

\section{References}

1. L. Adams, et al., "An Optical Navigator for Brain Surgery". IEEE Computer, 29(1):48-54, Jan. 1996.

2. D.R. Bucholtz, K.R. Smith. "A Comparison of Sonic Digitizers Versus Light Emitting Diode-Based Localization". R.J. Maciunas, ed., Interactive Image-Guided Neurosurgery, Amer. Assoc. Neur. Surg., 1993.

3. A. Chabrerie, F. Ozlen, S. Nakajima, M. Leventon, H. Atsumi, E. Grimson, E. Keeve, S. Helmers, J. Riviello, G. Holmes, F. Duffy, F. Jolesz, R. Kikinis, P. Black, "Three-Dimensional Reconstruction and Surgical Navigation in Pediatric Epilepsy Surgery", Medical Image Computation and Computer Assisted Interventions, Boston, October 1998.

4. P. Cinquin et al., "Computer Assisted Medical Interventions", IEEE EMB, 254263, May/June 1995.

5. A.C.F. Colchester et al., "Development and Preliminary Evaluation of VISLAN, a Surgical Planning and Guidance System Using Intra-Operative Video Imaging". Medical Image Analysis, 1(1):73-90, March 1996.

6. G. Ettinger, "Hierarchical three-dimensional medical image registration", Ph.D. Thesis, MIT, 1997.

7. G. Ettinger, et al., "Experimentation with a Transcranial Magnetic Stimulation System for Functional Brain Mapping", Medical Image Analysis, 2(2):133-142, 1998.

8. R. Galloway. "Stereotactic Frame Systems and Intraoperative Localization Devices". R. Maciunas, ed., Interactive Image-Guided Neurosurgery, Amer. Assoc. Neur. Surg., 1993.

9. W.E.L. Grimson, et al., "An Automatic Registration Method for Frameless Stereotaxy, Image Guided Surgery, and Enhanced Reality Visualization", Comp. Vis. and Pattern Recognition Conference, Seattle, June 1994. 
10. W.E.L. Grimson, et al., "Evaluating and Validating an Automated Registration System for Enhanced Reality Visualization in Surgery". First CVRMED, Nice France, April 1995, pp. 3-12.

11. W.E.L. Grimson, et al., "An Automatic Registration Method for Frameless Stereotaxy, Image Guided Surgery, and Enhanced Reality Visualization". IEEE TMI, 15(2):129-140, April 1996.

12. B. Horn. "Closed-form Solution of Absolute Orientation Using Unit Quaternions". JOSA A, 4:629-642, April 1987.

13. T. Kapur, W.E.L. Grimson, R. Kikinis. "Segmentation of Brain Tissue from MR Images". First CVRMED, Nice France, April 1995, pp. 429-433.

14. W.E. Lorensen, H.E. Cline. "Marching Cube: A High Resolution 3-D Surface Construction Algorithm". Computer Graphics, 21(3):163-169, 1987.

15. R. Maciunas, et al., "Beyond Stereotaxy: Extreme Levels of Application Accuracy Are Provided by Implantable Fiducial Markers for Interactive Image-Guided Neurosurgery". R.J. Maciunas, ed., Interactive Image-Guided Neurosurgery, Amer. Assoc. Neur. Surg., 1993.

16. T. McInerney, D. Terzopoulos. "Medical image segmentation using topologically adaptable snakes". CVRMed 1995.

17. T. Peters, et al., "Three-Dimensional Multimodal Image-Guidance for Neurosurgery". IEEE TMI, 15(2):121-128, April 1996.

18. M.J. Ryan, et al., "Frameless Stereotaxy with Real-Time Tracking of Patient Head Movement and Retrospective Patient-Image Registration". J. Neurosurgery, 85:287-292, August 1996.

19. G. Szekely, et al. "Segmentation of $3 \mathrm{~d}$ objects from MRI volume data using constrained elastic deformations of flexible fourier surface models". CVRMed 1995.

20. S. Tebo, et al., "An Optical 3D Digitizer for Frameless Stereotactic Surgery". IEEE Comp. Graph. Appl., 16(1):55-64, Jan. 1996.

21. K.L. Vincken, A.S.E. Koster, M.A. Viergever. "Probabilisitc hyperstack segmentation of MR Brain Data", CVRMed, 1995.

22. S. Warfield, et al., "Automatic identification of grey matter structures from MRI to improve the segmentation of white matter lesions". Journal of Image Guided Surgery 1(6):326-338, June 1996.

23. E. Watanabe, "The Neuronavigator: A Potentiometer-Based Localizing Arm System". R. Maciunas, ed., Interactive Image-Guided Neurosurgery, Amer. Assoc. Neur. Surg., 1993.

24. W.M. Wells. "Adaptive Segmentation of MRI Data". First CVRMED, Nice France, April 1995, pp. 59-69.

25. W.M. Wells, III, W.E.L. Grimson, R. Kikinis, F.A. Jolesz, "Adaptive Segmentation of MRI data", IEEE Trans. Medical Imaging, 15(4):429-442, August 1996.

26. W.M. Wells, et al. "Multi-modal volume registration by maximization of mutual information" Medical Image Analysis, 1(1): 35-51, 1996. 